\title{
Torque Ripple Minimization in PMSM Based on an Indirect Adaptive Robust Controller
}

\author{
Ruichao Tao, Jie Ma, and Hui Zhao \\ School of Astronautics, Harbin Institute of Technology, Heilongjiang, Harbin 150080, China \\ Correspondence should be addressed to Ruichao Tao; sliby7@163.com
}

Received 12 May 2017; Revised 5 August 2017; Accepted 23 August 2017; Published 1 October 2017

Academic Editor: Anna Vila

Copyright (c) 2017 Ruichao Tao et al. This is an open access article distributed under the Creative Commons Attribution License, which permits unrestricted use, distribution, and reproduction in any medium, provided the original work is properly cited.

\begin{abstract}
This paper addresses the problem of torque ripple minimization in permanent magnet synchronous motor (PMSM), which plays an important role in modern aerospace industry. Accurate motion control and disturbance compensation are challenging issues of PMSM systems, where the nonlinear disturbances are quite complicated and various uncertainties exist. To overcome these control problems, based on the adaptive robust control (ARC) algorithm, an indirect adaptive robust controller (IARC) with a robust recursive least square (RRLS) adaption law is proposed as a solution. A modified PMSM model which indicates the torque ripple generation is first derived. The IARC in current loop is then described, holding the good tracking performance of ARC algorithm and minimizing the torque ripples while speed tracking. The RRLS adaption law in IARC is synthesized based on modified model and then a correction factor is added to enhance the robustness of this adaptation law. This can enable the better parametric estimation and adaptive compensation to minimize the torque ripples. The stability of the system with the proposed controller is proved. Finally, the effectiveness of the proposed method is demonstrated by the simulation results.
\end{abstract}

\section{Introduction}

Permanent magnet synchronous motor (PMSM) has received much attention due to the inherent advantages of low rotor inertia, high efficiency, and high power density. It plays an important role in wide range of modern aerospace industry, such as satellite and electromechanical actuator [1-3]. The main disadvantage of PMSM is ripples in the generated torque which are not tolerated in these high performance applications. Torque ripples would lead to speed oscillations which cause deterioration in the servo performance of PMSM $[4,5]$. Thus, the key issue of control problem in PMSM focuses on ripple minimization.

Since the torque ripples mainly arise from nonideal backEMF in stators, a large number of techniques have been proposed for ripple minimization caused by this phenomenon. In [6], the off-line measurements of back-EMF of specific motor are used to calculate the optimal currents. Due to the off-line date, this technique is sensitive to parametric uncertainties. To account for parametric uncertainties, another effective method synthesizes an adaptive controller depending on the Lyapunov function of tracking error to estimate the harmonic parameters of back-EMF [7]. These estimated parameters are then used to calculate the current reference in time. In $[8,9]$, iterative learning control is applied in time domain and frequency domain, respectively, based on internal model principle. However, the additional torque sensors are costly in most application. An analytical model of torque is derived from magnetic coenergy by finite element analysis in [10]. A feedforward torque controller is then introduced for torque ripple reduction and maximum torque per ampere. Due to the finite element analysis, this analytical method is useful to the specified motors. These approaches above have not taken the nonlinear disturbances into account which limited the achievable performances of the algorithms.

Idealization of a mathematical model makes torque ripple minimization a tough challenge, such as the absence of uncertainties and disturbances. In [11], an adaptive robust control (ARC) algorithm for uncertain nonlinear system is proposed by Yao and Tomizuka. The ARC algorithm combines the parametric estimation of adaptive control with the robust stability of deterministic robust control. It was then applied 
to some industrial applications with different modifications. In [12], a $\mu$-synthesis-based adaptive robust controller was used to deal with the high-frequency dynamics for better disturbance rejection in linear motor driven system. The main effort is the design of a high-frequency robust control law, while the design of the adaptive law is same as [11]. To overcome the inherent nonlinear properties and uncertainties in hydraulic system, an adaptive robust controller with novel stable adaptive controller was introduced for stable estimation in [13]. Another adaptive robust controller combined with cascade control was proposed for human performance augmentation [14]. Meanwhile, many efforts are made for precision motion control in speed loop of PMSM systems as a direct adaptive robust controller (DARC) [1517]. However, being a direct Lyapunov design method based on tracking error, DARC does not provide the independent choice of adaption law. The adaption law is calculated by tracking error with the sole purpose to reduce the tracking error. While the actual tracking error in control loop is normally small, the adaptation law is prone to be corrupted and affected by other neglected factors. To overcome the poor estimated performance of the DARC, an indirect adaptive robust controller (IARC) with independent adaption law has been proposed [18]. Different from DARC, the adaption law of IARC is designed based on state formulas of physical plant. The separation of adaption law and robust control law provides more accurate parameter estimations. Such an independent adaption law is synthesized based on a filtered hydraulic system; the control law is still synthesized based on original model [19]. Since the models used in adaption design and control design are different, the stability analysis of IARC is limited.

In this paper, a novel IARC controller in current loop for minimizing torque ripples in PMSM systems is proposed. The controller is based on the same scheme as in [18]. To overcome the poor parameter estimation of DARC and complicated stability analysis, a robust recursive least square (RRLS) adaptation law is synthesized independent from the design of underlying robust control law. With the estimated harmonic parameters of back-EMF and a practical torque production model, the proposed IARC is designed in current loop to achieve ripple minimization control. In addition, a PI controller is introduced in speed loop.

The remainder of this paper is organized as follows. In Section 2, a PMSM model with nonideal back-EMF is derived. This model is then transformed into state space form for the design of IARC. The prosed control scheme for torque ripple minimization, which includes an IARC in current loop and a PI controller in speed loop, is introduced in Section 3. Compared with the DARC, a RRLS adaptation law is synthesized according to the state equations. In Section 4, simulation results supporting the theoretical derivations are presented.

\section{Dynamic Model and Problem Formulation}

A brief discussion about torque production in PMSM is presented in this section. A PMSM model with nonideal back-EMF is developed for analysis and controller design.

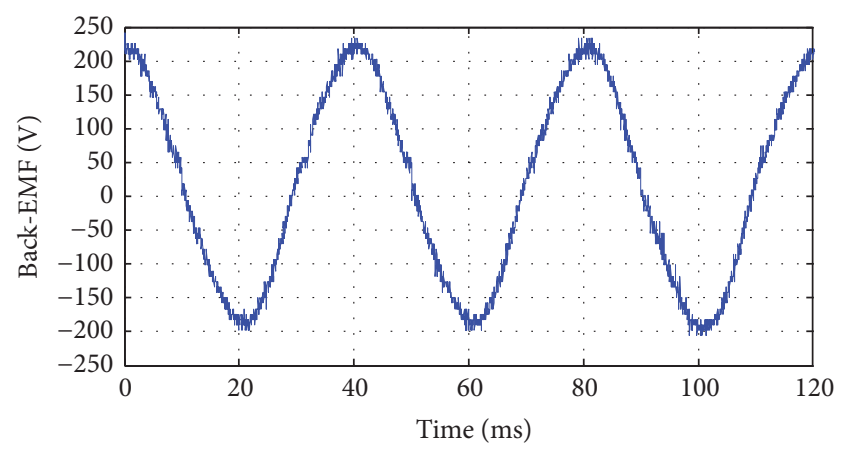

FIGURE 1: Measured back-EMF in one phase of an experimental PMSM.

The mutual interaction of the rotor filed and stator current produces torque in PMSM. Ideally, the rotated rotor filed generated by permanent magnet in air gap is purely sinusoidal and induced in stators as the back-EMF. The ideal back-EMF would be a constant value in synchronous frame and then produce a constant electromagnetic torque. As the cogging structure design for PMSM, the ideal sinusoidal field is not achievable in practice. This factor can contribute to high harmonics in torque, that is, torque ripples. The torque ripples appear at multiples of 6 fundamental electronic frequencies which indicate the existence of $6 n$ harmonics in back-EMF [20]. Figure 1 shows the back-EMF of an experimental PMSM, related to a unitary speed.

The conventional vector control strategy for current loop is applied with PI controller based on the ideal $d q$ model of PMSM. With the purpose of the minimization of torque ripples, a modified $d q$ model is developed considering the nonideal back-EMF, which is also compact and suitable for controller design. Some reasonable assumptions for the modified model and controller design are made as follows.

Assumption 1. The type of PMSM in this paper is surfacemounted, which means the influences of nonlinear features of inductances can be ignored.

Assumption 2. The uncertainties of nonideal back-EMF and the disturbances in this derived model are bounded and the boundaries are known.

Assumption 3. An approximate model of nonideal backEMF is proposed consisting of fundamental and 6 harmonic components, and the residual approximate error can be regarded as part of the disturbances.

Assumption 4. The cogging torque is suppressed by skewed slots or other manufacturing procedure.

Under above assumptions, the modified PMSM model is

$$
\begin{aligned}
L \frac{\mathrm{d}}{\mathrm{d} t}\left[\begin{array}{l}
i_{d} \\
i_{q}
\end{array}\right]= & {\left[\begin{array}{l}
u_{d} \\
u_{q}
\end{array}\right]-R\left[\begin{array}{l}
i_{d} \\
i_{q}
\end{array}\right]+\omega L\left[\begin{array}{cc}
0 & 1 \\
-1 & 0
\end{array}\right]\left[\begin{array}{l}
i_{d} \\
i_{q}
\end{array}\right] } \\
& -\left[\begin{array}{l}
e_{d} \\
e_{q}
\end{array}\right]+\left[\begin{array}{l}
\Delta_{d} \\
\Delta_{q}
\end{array}\right],
\end{aligned}
$$




$$
\begin{gathered}
T_{e}=\frac{i_{d} e_{d}+i_{q} e_{q}}{\omega}, \\
J \dot{\omega}=T_{e}-B \omega-T_{l},
\end{gathered}
$$

where $L$ and $R$ are stator inductances and resistances in $d q$ frame. $i_{d}$ and $i_{q}$ are currents in stators. $u_{d}$ and $u_{q}$ are control variables. $e_{d}$ and $e_{q}$ are back-EMFs produced by rotated rotor field. $T_{e}$ is torque produced by the PMSM and $T_{l}$ is a load torque. $J$ and $B$ are the inertia and the friction constant. $\omega$ is motor speed in $\mathrm{rad} / \mathrm{s} . \Delta_{d}$ and $\Delta_{q}$ are the lumped disturbances in $d q$ frame.

With the similar form as conventional model, the essential differences between them are the back-EMF terms in (1) and the consequent torque expression (2). A back-EMF expression considering the nonideal feature is

$$
\left[\begin{array}{c}
e_{d} \\
e_{q}
\end{array}\right]=\frac{3}{2} \omega_{e}\left[\begin{array}{c|cc}
\sin \left(6 \theta_{e}\right) & 0 & 0 \\
\hline 0 & 1 & \cos \left(6 \theta_{e}\right)
\end{array}\right]\left[\begin{array}{c}
K_{d 6} \\
K_{q 1} \\
K_{q 6}
\end{array}\right],
$$

where $\theta_{e}$ and $\omega_{e}$ are electric position and speed. $K_{d 6}, K_{q 1}$, and $K_{q 6}$ are harmonic parameters of back-EMF in $d q$ frame. Due to the harmonics in (4), the torque expression cannot be formed as a constant torque coefficient multiplied by the $\omega_{e}$ [21].Therefore, the formula (2) is given on account of nonideal back-EMF by the mechanism of torque production in PMSM.

\section{Proposed Control Scheme}

In this section, a two loop control scheme is designed for torque ripples minimization with an indirect adaptive robust current controller and a typical PI speed controller. An IARC in current loop is proposed focusing on the compensation of the ripples caused by nonideal back-EMF. Since the ripples have been minimized in current loop, a PI controller designed in speed loop is enough.

3.1. Indirect Adaptive Robust Controller in Current Loop. With a priori knowledge on the bounds of unknown parameter in Assumption 1, regardless of the specific adaption law to be used, the estimation errors of unknown parameters are bounded by some known values even in the presence of disturbances and uncertainties. Therefore, an IARC can be synthesized in accordance with these known values for the system (1).

For the convenience of current controller design, a virtual control $u_{i}$ consisting of known and measurable variables is introduced into (1) and the model (1) can be rewritten as the standard form as

$$
L \dot{x}=u_{i}+\varphi^{\mathrm{T}} \theta+\Delta
$$

where

$$
\begin{aligned}
x & =\left[\begin{array}{l}
i_{d} \\
i_{q}
\end{array}\right], \\
u_{i} & =\left[\begin{array}{l}
u_{i_{d}} \\
u_{i_{-q}}
\end{array}\right]=\left[\begin{array}{l}
u_{d} \\
u_{q}
\end{array}\right]-R\left[\begin{array}{l}
i_{d} \\
i_{q}
\end{array}\right]+\omega L\left[\begin{array}{cc}
0 & 1 \\
-1 & 0
\end{array}\right]\left[\begin{array}{l}
i_{d} \\
i_{q}
\end{array}\right],
\end{aligned}
$$

$$
\begin{aligned}
\varphi^{\mathrm{T}} & =\left[\begin{array}{c|c}
\varphi_{d}^{\mathrm{T}} & 0 \\
\hline 0 & \varphi_{q}^{\mathrm{T}}
\end{array}\right]=\frac{3}{2} \omega_{e}\left[\begin{array}{c|cc}
\sin \left(6 \theta_{e}\right) & 0 & 0 \\
\hline 0 & 1 & \cos \left(6 \theta_{e}\right)
\end{array}\right] \\
\theta & =\left[\begin{array}{c}
\theta_{d} \\
\hline \theta_{q}
\end{array}\right]=\left[\begin{array}{l}
\frac{K_{d 6}}{K_{q 1}} \\
K_{q 6}
\end{array}\right], \\
\Delta & =\left[\begin{array}{l}
\Delta_{d} \\
\Delta_{q}
\end{array}\right],
\end{aligned}
$$

The state variable $x$ is defined as the currents of $d q$ frame. $\varphi^{\mathrm{T}}$ is a known function and $\theta$ is a set of unknown parameters.

According to assumptions, the unknown parameter vector $\theta$ and all the disturbances $\Delta$ lie within a known bounded set as

$$
\begin{aligned}
& \theta \in \Omega_{\theta}=\left\{\theta: \theta_{\min } \leq \theta \leq \theta_{\max }\right\}, \\
& \Delta \in \Omega_{\Delta}=\left\{\Delta:\left|\Delta_{d}\right| \leq \Delta_{m},\left|\Delta_{q}\right| \leq \Delta_{m}\right\},
\end{aligned}
$$

where $\theta_{\min }$ and $\theta_{\max }$ are known lower and upper bound vectors of $\theta$ and $\Delta_{m}$ is a known value. For simplification, we can let $\theta_{\min }=-\theta_{\max }$ in this section.

Based on the reformed model (5), a control law $u_{i}$ of IARC which contains the features of adaptive control and deterministic robust control can be synthesized as

$$
u_{i}=u_{m}+u_{s}
$$

where $u_{m}$ and $u_{s}$ are the model adaptive compensation term and robust feedback control term respectively:

$$
\begin{aligned}
& u_{m}=L \dot{x}_{d}-\varphi^{\mathrm{T}} \hat{\theta}, \\
& u_{s}=u_{s 1}+u_{s 2}, \quad u_{s 1}=-k_{s 1} z_{i},
\end{aligned}
$$

where

$$
\begin{aligned}
& x_{d}=\left[\begin{array}{l}
i_{d_{\text {ref }}} \\
i_{q_{\text {ref }}}
\end{array}\right], \\
& z_{i}=\left[\begin{array}{l}
z_{i_{-}} \\
z_{i_{-q}}
\end{array}\right], \\
& k_{s 1}=\left[\begin{array}{cc}
k_{s 1 \_d} & 0 \\
0 & k_{s 1 \_q}
\end{array}\right], \\
& u_{s 2}=\left[\begin{array}{l}
u_{s 2 \_d} \\
u_{s 2 \_q}
\end{array}\right],
\end{aligned}
$$

where $\hat{\theta}$ is the estimation of $\theta$ by an adaption law designed later. $x_{d}$ is the desired trajectory and $z_{i}$ is the tracking error of current, $z_{i}=x_{d}-x . u_{s 1}$ is a linear feedback control term and $u_{s 2}$ is a robust control term. In (10), $u_{s 2}$ is designed to overcome all types of model uncertainties and disturbances to achieve robust stability and certain robust performance. 


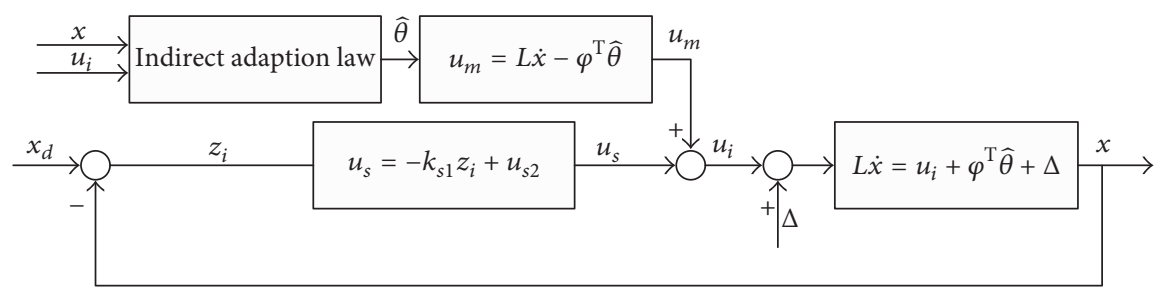

Figure 2: Scheme of IARC.

The deterministic robust control term $u_{s 2}$ should satisfy the following two conditions [11]:

$$
\begin{aligned}
& z_{i_{-} \cdot} u_{s 2_{-} \cdot} \leq 0, \\
& z_{i_{-} \cdot}\left\{u_{s 2_{-}}-\left[\varphi_{\bullet}^{\mathrm{T}} \tilde{\theta}_{\bullet}-\Delta . \cdot\right]\right\} \leq \varepsilon_{\bullet},
\end{aligned}
$$

where the subscript "•" is used to represent $d$ or $q . \tilde{\theta}$ is the estimation error of unknown parameter and $\varepsilon_{M}=\left[\varepsilon_{d}, \varepsilon_{q}\right]$ is a specified value.

For mathematical convenience, a satisfied choice of $u_{s 2}$ is

$$
u_{s 2}=-E H z_{i}
$$

where

$$
\begin{aligned}
& h=\left|\varphi^{\mathrm{T}}\right|\left|\theta_{\max }-\theta_{\min }\right|+\Delta_{m}=\left[\begin{array}{l}
h_{d} \\
h_{q}
\end{array}\right], \\
& E=\frac{1}{4} \operatorname{diag}\left\{\frac{1}{\varepsilon_{d}}, \frac{1}{\varepsilon_{q}}\right\}, \\
& H=\operatorname{diag}\left\{h_{d}^{2}, h_{q}^{2}\right\} .
\end{aligned}
$$

Combined with the IARC (8), the dynamic equation of tracking error is

$$
L \dot{z}_{i}-u_{s 1}=u_{s 2}-\left[\varphi^{\mathrm{T}} \widetilde{\theta}-\Delta\right]
$$

From (15), the stability performance of current loop is mainly determined by the design of robust control term $u_{s}$. Therefore, a reasonable $u_{s}$ of IARC can preserve the nice tracking performance of DARC. Moreover, due to the separation of control law and adaption law in IARC, an indirectly adaption law can achieve better parameter estimation for compensation or diagnosis purpose.

By the control law (8), the scheme of IARC is shown in Figure 2.

\subsection{Indirect Adaptive Law Based on Robust Recursive Least} Squares Algorithm. The main advantage of the IARC design is to achieve complete separation of adaptation law design and robust control law design. Without using the tracking error to synthesize the adaptation law, an observation model and an estimation model are constructed by practical information of state formulas (5). By doing this, more accurate and stable estimation of uncertain parameters can be obtained even in the presence of disturbance.
For making this expression clear, we first discuss the design of an adaption law of $q$ axis. Based on (5), the observation model and estimation model of $q$ axis are as follows:

$$
\begin{aligned}
& y_{q}(k)=L \dot{i}_{q-\text { ref }}(k)-u_{i_{d}}(k)=\varphi_{q}^{\mathrm{T}} \theta_{q}+\Delta_{q}, \\
& \widehat{y}_{q}(k)=\varphi_{q}^{\mathrm{T}} \widehat{\theta}_{q}(k),
\end{aligned}
$$

where $y_{q}(k)$ is calculated by the known virtual control term and measurable states and $k$ means the sampling time.

By (16), the model estimation error is given as

$$
e=y_{q}-\widehat{y}_{q}=\varphi_{q}^{\mathrm{T}} \tilde{\theta}_{q}+\Delta_{q}
$$

A typical least squares adaptation law can be designed by (17) $[22,23]$. The disturbance $\Delta_{q}$ in (17) indicates that the model estimation error converging to zero is not equal to the parametric estimation error converging to zero any more. Therefore, a robust least squares algorithm is proposed and the iteration form is derived for its application in practical servo control.

Similar to the process of designing a least squares algorithm, a novel optimal function of estimation error $e$ with a compensation factor $\lambda_{q}$ is

$$
\min J\left(\widehat{\theta}_{q}\right)=\min \left\{\left\|\Phi_{q} \widehat{\theta}_{q}-Y_{q}\right\|_{2}^{2}+\lambda_{q}\left\|\widehat{\theta}_{q}\right\|_{2}^{2}\right\} .
$$

The additional second term $\lambda_{q}\left\|\widehat{\theta}_{q}\right\|_{2}^{2}$ in (18) is used as compensation for $\Delta_{q}$ in (17). The $\Phi_{q}$ and $Y_{q}$ are vectors consisting of measurable values and calculated values in (16) as

$$
\begin{gathered}
\Phi_{q}=\left[\begin{array}{c}
\varphi_{q}^{\mathrm{T}}(1) \\
\cdots \\
\varphi_{q}^{\mathrm{T}}(k)
\end{array}\right], \\
Y_{q}=\left[\begin{array}{c}
y_{q}(1) \\
\cdots \\
y_{q}(k)
\end{array}\right] .
\end{gathered}
$$

The solution of (18) is

$$
\widehat{\theta}_{q}=\left(\Phi_{q}^{\mathrm{T}} \Phi_{q}+\lambda_{q} I\right)^{-1} \Phi_{q}^{\mathrm{T}} Y_{q}
$$

The form of (20) is robust least square estimation. Inspired by the recursive least square algorithm, a recursive form of (20) which is suitable for practical application can be derived. 
As expressed in recursive least squares algorithm, let us define an intermediate variable as follows:

$$
Q_{q}^{-1}(k)=\Phi_{q}^{\mathrm{T}}(k) \Phi_{q}(k)+\lambda_{q} I=\sum_{i=1}^{k} \varphi_{q}(i) \varphi_{q}^{\mathrm{T}}(i)+\lambda_{q} I .
$$

By the matrix inversion lemma, an approximation recursive form of (21) can be given as [24]

$$
Q_{q}(k+1)=\left(I-\frac{Q_{q}(k) \varphi_{q}(k) \varphi_{q}^{\mathrm{T}}(k)}{1+\varphi_{q}^{\mathrm{T}}(k) Q_{q}(k) \varphi_{q}(k)}\right) Q_{q}(k)
$$

The value of compensation factor in RRLS algorithm is another issue in estimation process. In this paper, the coefficients of nonideal back-EMF can be regarded as constant or slowly changed. Thus, a concise method is to take the compensation effort as fixed variable. The $\lambda_{q}$ in (18) can be calculated by Morozov's discrepancy principle based on a set of off-line data [25]:

$$
\frac{1}{2}\left\|\Phi^{\mathrm{T}} \hat{\theta}_{q}-Y_{q}\right\|^{2}+\frac{1}{2} \lambda_{q}\left\|\hat{\theta}_{q}\right\|^{2} \leq \Delta_{q} .
$$

Therefore, the RRLS algorithm for $q$ axis is

$$
\begin{aligned}
\hat{\theta}_{q}(k+1)= & {\left[I-\lambda_{q} Q_{q}(k)\right] \hat{\theta}_{q}(k) } \\
& +K_{q}(k)\left[y_{q}(k)-\varphi_{q}^{\mathrm{T}}(k) \hat{\theta}_{q}(k)\right],
\end{aligned}
$$

where

$$
\begin{aligned}
& K_{q}(k+1)=\frac{Q_{q}(k) \varphi_{q}(k+1)}{\varphi_{q}^{\mathrm{T}}(k+1) Q_{q}(k) \varphi_{q}^{\mathrm{T}}(k+1)+1}, \\
& Q_{q}(k+1)=\left(I-K_{q}(k+1) \varphi_{q}^{\mathrm{T}}(k+1)\right) Q_{q}(k) .
\end{aligned}
$$

In a similar way, we can obtain the adaption law of $d$ axis:

$$
\begin{aligned}
\widehat{\theta}_{d}(k+1)= & {\left[1-\lambda_{d} Q_{d}(k)\right] \widehat{\theta}_{d}(k) } \\
& +K_{d}(k)\left[y_{d}(k)-\varphi_{d}^{\mathrm{T}}(k) \hat{\theta}_{d}(k)\right],
\end{aligned}
$$

where

$$
\begin{aligned}
K_{d}(k+1) & =\frac{Q_{d}(k) \varphi_{d}^{\mathrm{T}}(k+1)}{\varphi_{d}^{\mathrm{T}}(k+1) Q_{d}(k) \varphi_{d}^{\mathrm{T}}(k+1)+1}, \\
Q_{d}(k+1) & =\left(1-K_{d}(k+1) \varphi_{d}^{\mathrm{T}}(k+1)\right) Q_{d}(k) .
\end{aligned}
$$

Based on Assumption 1, to keep the boundedness of estimated parameters even in the presence of impulse disturbance, a projection mapping $\operatorname{Proj}(*)$ is used as

$$
\operatorname{Proj}(\widehat{\theta})= \begin{cases}\hat{\theta}, & \theta_{\min } \leq \hat{\theta} \leq \theta_{\max }, \\ \theta_{\max } \operatorname{sign}\left(\widehat{\theta}_{R}\right), & \widehat{\theta}>\theta_{\text {max }}, \\ \theta_{\text {min }} \operatorname{sign}\left(\hat{\theta}_{R}\right), & \widehat{\theta}<\theta_{\text {min }},\end{cases}
$$

where $\operatorname{sign}(*)$ means the sign function.

Hence, we have the adaption law as follows:

$$
\widehat{\theta}=\operatorname{Proj}(\widehat{\theta}) \text {. }
$$

\subsection{Performance of IARC}

Theorem 5. Under the assumptions, with an adaption law (29) and an indirect adaptive robust control law (8), all the signals in the closed-loop system are bounded and the following properties hold.

(1) In general, the tracking error has a guaranteed transient performance and final tracking accuracy. Meanwhile, the estimation error is bounded with a known value.

(2) If the parametric uncertainties existed only after a finite time, the tracking error asymptotically converges to zero and the estimated parameters converge to the optimal estimations.

Proof. For making this expression clear, we first discuss the stability analysis of IARC in $q$ axis. The proof results of $q$ axis are the same as $d$ axis. However, the control variable $u_{i}$ consisting of $u_{d}$ and $u_{q}$ can be synthesized at the same time.

Based on the dynamic equation of tracking error (15), letting the nonnegative Lyapunov function $V=\left(L z_{i_{-}}^{2}\right) / 2$, we have

$$
\begin{aligned}
\dot{V} & =L \dot{z}_{i_{-q}} z_{i_{-q}} \\
& =-k_{s 1-q} z_{i_{-q}}^{2}+z_{i_{-q}}\left[u_{s 2-q}-\left(\varphi_{q}^{\mathrm{T}} \widetilde{\theta}_{q}\right)+\Delta_{q}\right] .
\end{aligned}
$$

Noting (12), the derivative of $V$ satisfies

$$
\dot{V} \leq-k_{s 1_{-q}} z_{i_{-} q}^{2}+\varepsilon_{q} \leq-2 k_{s 1_{-q}} V+\varepsilon_{q} .
$$

Applying the Comparison Lemma, we have

$$
\begin{aligned}
\left|z_{i_{-} q}(t)\right|^{2} \leq & \left|z_{i_{-q}}(0)\right|^{2} \exp \left(-2 k_{s 1_{-} q} t\right) \\
& +\frac{\varepsilon_{q}}{k_{s 1_{-q}}}\left[1-\exp \left(-2 k_{s 1_{-} q} t\right)\right] .
\end{aligned}
$$

The result (1) is proved.

Furthermore, if the disturbances in observation model are zero, we have $\lambda_{q}=0$ in Morozov's discrepancy principle. The RRLS adaption law converts to RLS type. Therefore, the parameters of RLS converge to their optimal estimation. Meanwhile, the total disturbance $\left(\varphi^{\mathrm{T}} \widetilde{\theta}_{R}\right)+\Delta=0$ leads to $u_{s 2_{-} q}$ $=0$.

The dynamic error equation is turned into

$$
\dot{z}_{i_{-q}}+k_{s 1_{-}} z_{i_{-q}}=0
$$

Obviously, the tracking error $z_{i_{-} q}$ converges to zero asymptotically.

The result (2) is proved.

3.4. PI Controller in Speed Loop. The proposed control law (8) guarantees the perfect tracking of current reference from a speed controller and estimation of parameters in nonideal back-EMF. Since the proposed IARC combines the adaptive feedback linear part with the model of PMSM in current loop, the generalized control plant of speed loop can be recognized 


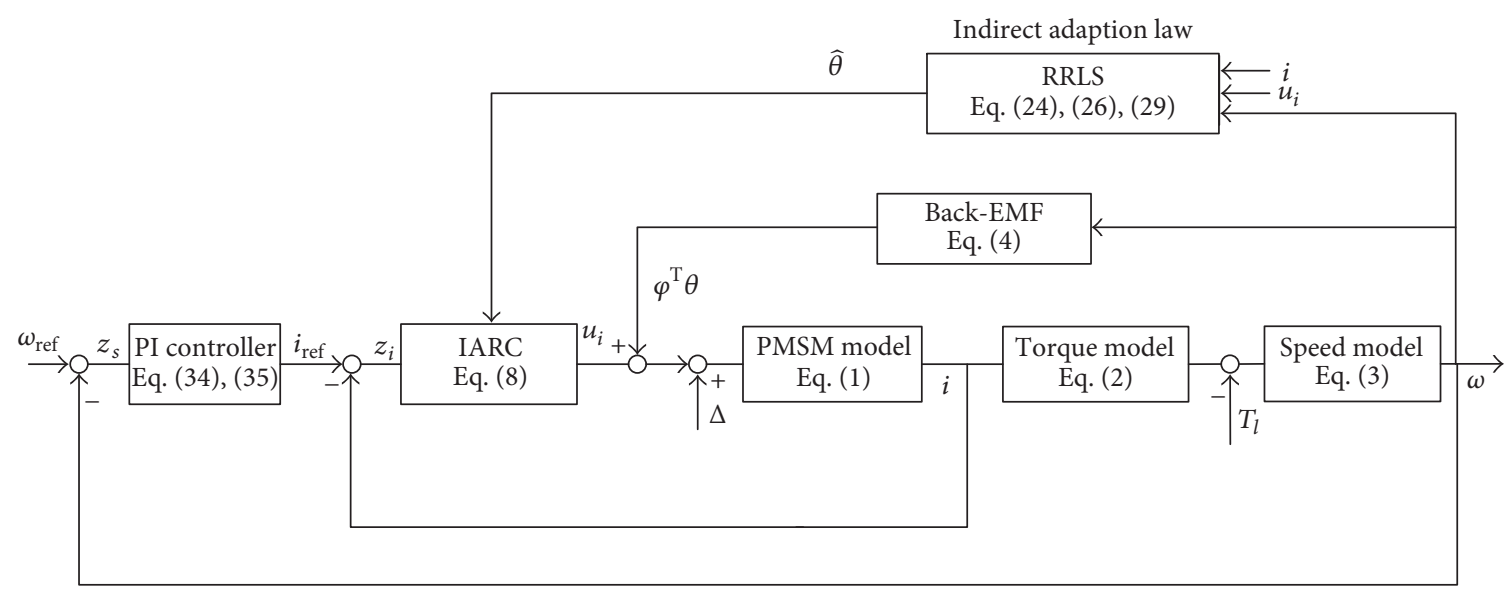

FIgURE 3: Scheme of torque minimization based on IARC.

as a linear model. Therefore, a feasible way to achieve speed servo operation in practice is to design a PI controller with the tracking error of speed as input.

$$
u_{r}(t)=k_{p} z_{s}(t)+k_{i} \int z_{s}(t) \mathrm{d} t,
$$

where $u_{r}$ is output of controller. $z_{s}$ is tracking error of speed decided by desired reference and feedback signal of speed loop. $k_{p}$ and $k_{i}$ are the proportional and integral coefficients of PI controller, respectively.

Although a steady error may exist in the current loop from result (1) due to the nonzero specified value $\varepsilon_{M}$ and finite linear feedback gain $k_{s 1}$, this steady error of current loop has a little influence on the output performance of PMSM which can be eliminated by the PI controller in speed loop.

This PI controller generates desired torque reference $T_{e}$ ref according to the desired speed reference in (3). Due to the absence of dc component in $e_{d}$, a nonzero $i_{d}$ generates only torque ripples which is not tolerant in most servo systems. Thus, it is desirable to set $i_{d \text { ref }}$ equal to zero and the desired current is transformed from $T_{e_{-} \text {ref }}$ as

$$
i_{\text {_ref }}=\left[\begin{array}{c}
i_{d_{\_} \text {ref }} \\
i_{q_{-} \text {ref }}
\end{array}\right]=\left[\begin{array}{c}
0 \\
\frac{T_{e_{-} \text {ref }}}{\hat{e}_{q}}
\end{array}\right] .
$$

From the above, the control scheme of the propose torque minimization method is shown in Figure 3.

\section{Simulation and Analysis}

After the theoretical derivation, the proposed torque minimization method was implemented in simulation. In addition, we also gave some simulation results of IARC to prove the correctness of the proposed controller. The PMSM in simulation and experiment is the same one shown in Figure 1. Parameters of PMSM are shown in Table 1.

4.1. Simulation Results of IARC. First, an IARC was implemented in MATLAB with a simplification to verify the accuracy of the proposed adaption law and tracking performance.
TABLE 1: Motor specifications.

\begin{tabular}{lc}
\hline Parameters and symbols & Value \\
\hline Rated voltage $U$ & $380 \mathrm{~V}$ \\
Rated power $P_{n}$ & $8 \mathrm{kw}$ \\
Number of pole pairs $p$ & 10 \\
Stator resistance $R$ & $0.504 \Omega$ \\
Stator inductance $L$ & $7.1 \mathrm{mH}$ \\
Rotational inertia $J$ & $0.2 \mathrm{Kg} \cdot \mathrm{m}^{2}$ \\
\hline
\end{tabular}

The controller was simulated in $q$ axis without taking into account any effects of speed loop and $d$ axis. Let $i_{q-\text { ref }}=1.5 \mathrm{~A}$, and rotor speed $\omega=10 \mathrm{rad} / \mathrm{s}$. The "true" value of unknown parameters is set as $[2,0.5]$. The parameters of PI controller, DARC, and IARC are as follows:

(1) PI controller: proportionality parameter $k_{p}=0.3$, and integral parameter $k_{i}=0.02$

(2) DARC [6]: for simplification, deterministic robust control term $u_{s 2_{-q}}=-\left(h_{q}^{2} / \varepsilon_{q}\right) z_{i_{-q}}$ can be written as $u_{s 2_{-} q}=k_{s 2_{-} q_{i_{-}}}$. Let $u_{s_{-} q}=-k_{s_{-} q} z_{i_{-q}}, k_{s_{-} q}=k_{s 1_{-} q}+$ $k_{s 2_{-} q}=13$. The lower and upper bounds of unknown parameter vector $\theta_{q}$ are set as $[-3,-0.8]$ and $[3,0.8]$. The initial value of these parameters is $[0,0.1]$. The disturbance is set as $\Delta=\operatorname{rand}(1)$ which is bounded within a known value.

(3) IARC: to have a comparison, controller parameters and initial values of unknown parameters were kept the same for both DARC controller and IARC. The compensation factor was set as $\lambda_{q}=2$, and the initial value of matrix $Q$ was $Q(0)=1000 I$.

Figure 4 shows the tracking performance of IARC comparing with other two controllers. As seen from Figure 4, ARC, which can handle the harmonics and nonlinear disturbances effectively, has better tracking performance than common PI controller. The current waveform of ARC is smooth while the convergence is fast. As seen from this picture, due to the existence of disturbance in observation 


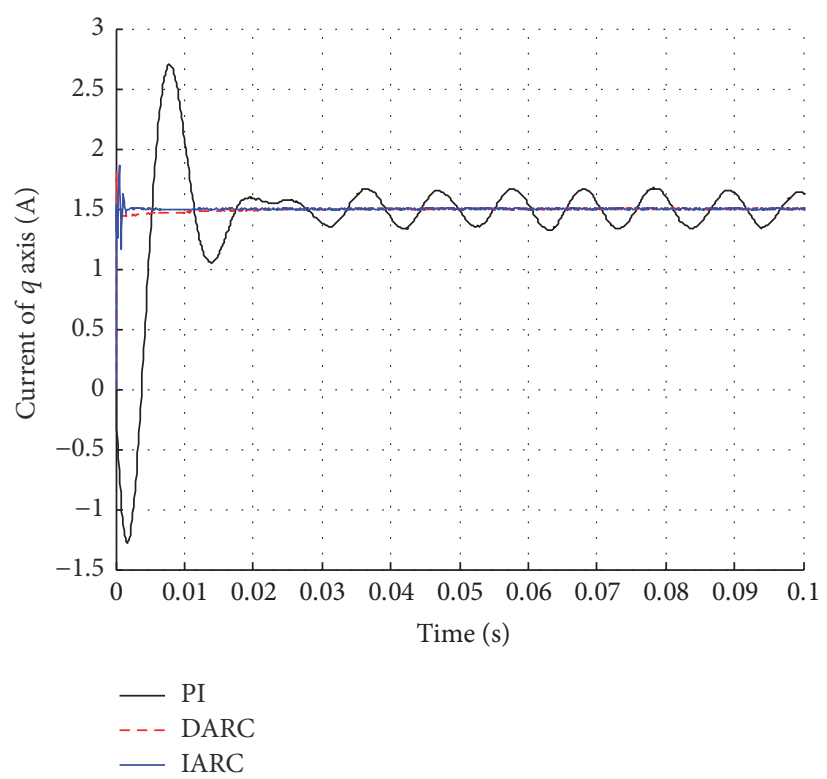

FIGURE 4: Tracking performances of comparative controllers.

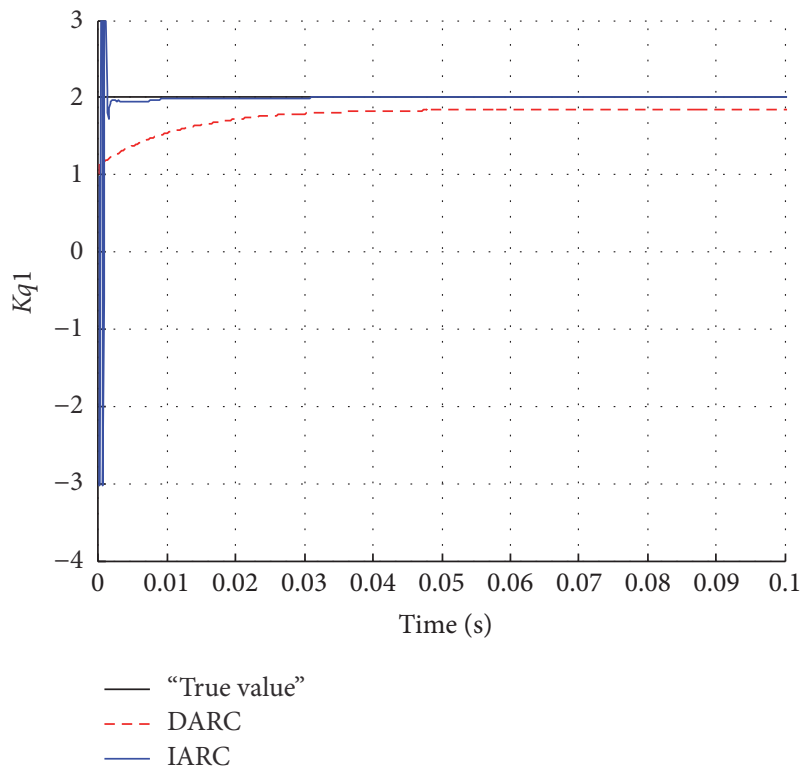

(a) Estimation of $K_{q 1}$

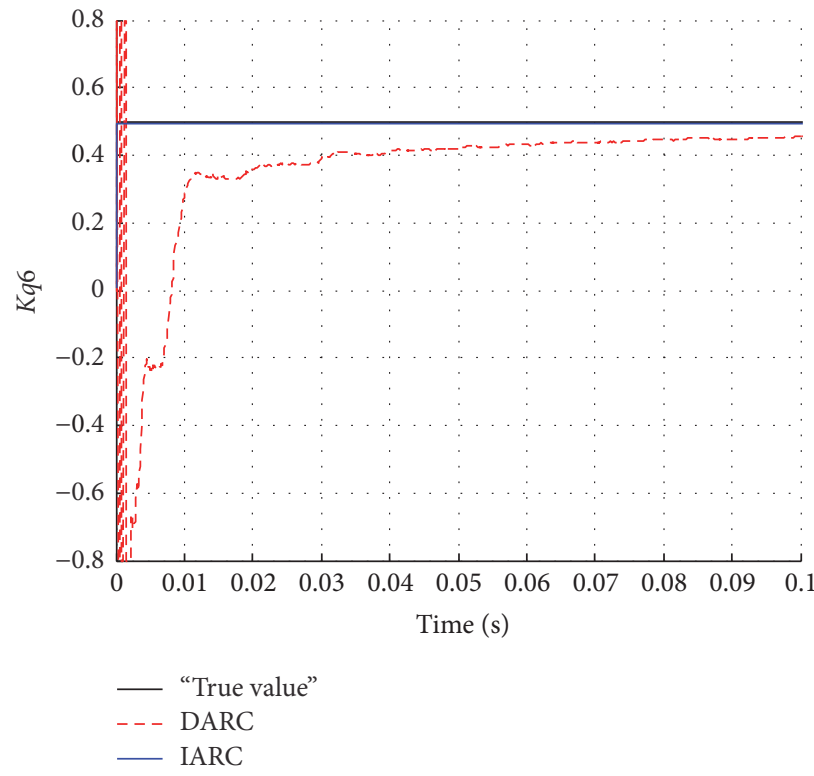

(b) Estimation of $K_{q 6}$

FIgURE 5: Estimated parameters of DARC and IARC.

model, IARC has better convergence rate and compensation than DARC. The accuracy of these unknown parameter estimated by IARC and DARC can be seen in Figure 5 . Since the adaption law in DARC is designed corresponding to tracking error, the estimated convergence rate of DARC is synchronous with tracking error and slower than IARC. In addition, the estimated error of DARC is larger than IARC due to the existence of disturbance which was ignored in DARC. Thus, a better adaptive compensation can be obtained by IARC. The relatively more accurate parameter estimations of IARC may also be used for other secondary purposes, such as diagnoses. We can also see that the projection mapping guarantees the boundary of all estimated parameters.

4.2. Simulation Results of Torque Minimization Method. Based on the scheme shown in Figure 3, an integrated simulation model was formed in MATLAB to verify the effectiveness of proposed torque minimization control in this paper. The speed reference is $\omega_{\text {ref }}=5 \mathrm{rad} / \mathrm{s}$. The "true" value of unknown parameters is set as $[0.5,2,0.5]$. An adaptive controller (AC) for torque minimization is introduced as a comparison [7]. Since the adaptive controller is always 


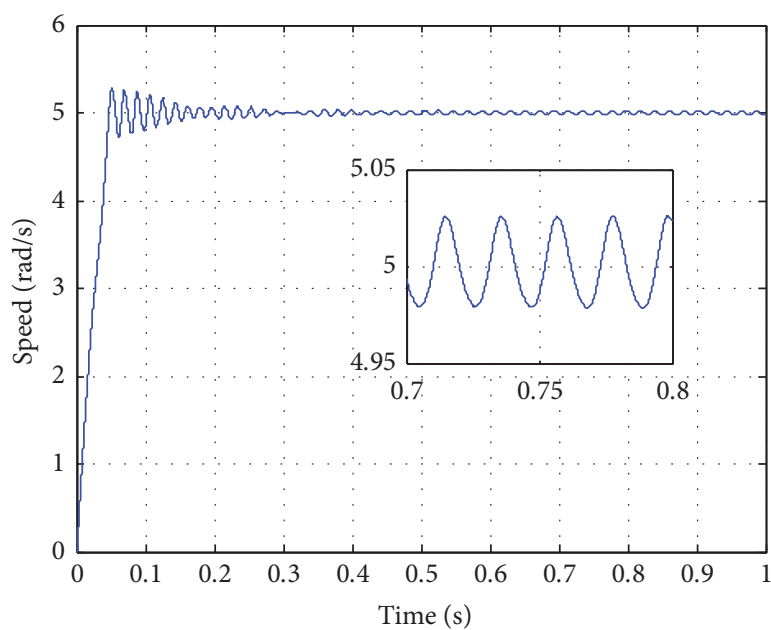

(a) Speed waveforms before torque ripple minimization

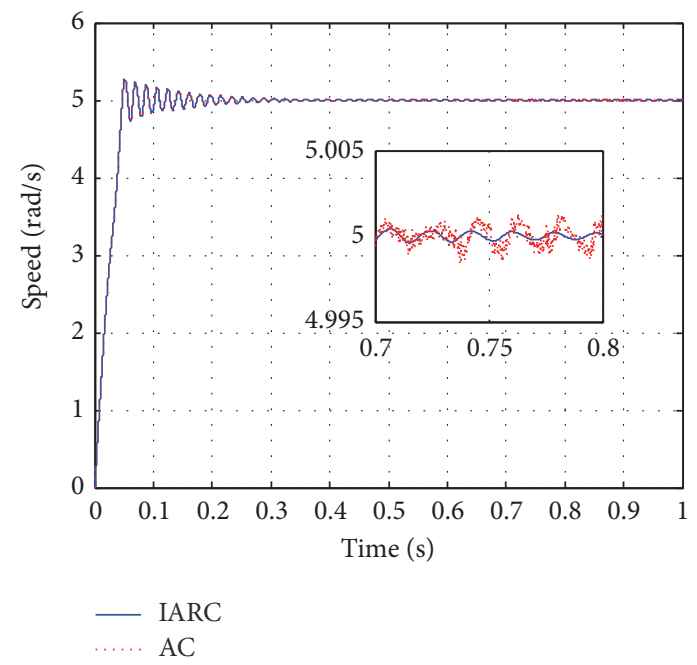

(b) Speed waveforms after torque ripple minimization

FIGURE 6: Speed waveforms before and after torque minimization.

derived from Lyapunov design method, which is similar to $u_{m}$ in DARC, an adaptive controller with similar expression is used. The parameters of PI controller in speed loop, IARC, and adaptive controller in current loop are set as follows:

(1) PI controller in speed loop: $k_{p_{-} s}=20$, and $k_{i_{-} s}=1.2$.

(2) IARC in current loop: The lower and upper bounds of unknown parameter vector are set as $[-3,-1,-1]$ and $[3,1,1]$. The initial value of these parameters is $[0.1,0,0.1]$. As in Section 4.1, let control gain matrix be set as $k_{s}=\operatorname{diag}\{5,13\}$. The compensation factor is $\lambda_{d}=\lambda_{q}=2$, and the initial values of matrixes are $Q_{d}(0)=Q_{q}(0)=1000 I$.

(3) AC in current loop: let $u_{\mathrm{ac}}$ be the control law of adaptive control:

$$
u_{\mathrm{ac}}=L \dot{x}_{d}-\varphi^{\mathrm{T}} \hat{\theta}
$$

where

$$
\dot{\hat{\theta}}=\operatorname{Proj}\left(\Gamma \varphi z_{i}\right)
$$

The $\Gamma$ is the adaptive rate matrix; $\Gamma$ is chosen based on a trade-off between speed and oscillations in parameter convergence; the function Proj( ) has the same form as (28). Other parameters are the same as those in IARC.

The performance of proposed torque ripple minimization method in this paper is shown in Figures 6-8. As shown in Figure 6 , the speed converges to the steady state with obvious ripples before torque ripple minimization. The ripples were mainly caused by the nonideal back-EMF. After the proposed method and AC being implemented, the ripples in speed were decreased. Compared with the results of adaptive controller, the IARC obtains better performance of torque minimization which considers the disturbances in model. Since the adaptive compensation was only consisted of fundamental and 6 harmonic components, there were still little ripples in current as shown in Figure 7(b). However, these ripples in current are so small to make little influence in speed as shown in Figure 6. Figure 8 shows the estimated results of IARC. The current tracking performances between IARC and adaptive controller are similar as shown in Figure 4. The RRLS adaption law in IARC is designed based on state formulas. As seen from this figure, the estimated parameters converged to the "true" value with fast rate. Different from DARC, the estimated parameters converged before the tracking error converged to steady state. Since the parameter estimations approach their "true" values, IARC achieves good adaptive compensation performance.

\section{Conclusions}

This paper concerns the torque ripple minimization problem caused by nonideal back-EMF in PMSM. Based on the mechanism that in the PMSM the torque is generated by the mutual interaction of the rotor filed and stator current, a modified model with uncertainties and disturbances is derived. Due to the nonlinear disturbances and various uncertainties in PMSM model, a novel indirect adaptive robust controller is proposed with a robust recursive least squares adaption law in current loop. The proposed IARC focuses on accurate estimations of unknown parameters. Therefore, the influence of disturbances can be compensated. The tracking and estimated performance of the proposed controller is analyzed. Simulation results show a significant merit of the proposed controller, that is, better steadystate tracking performance and more accurate parametric estimation in comparison with common PI controller or direct adaptive robust controller. 

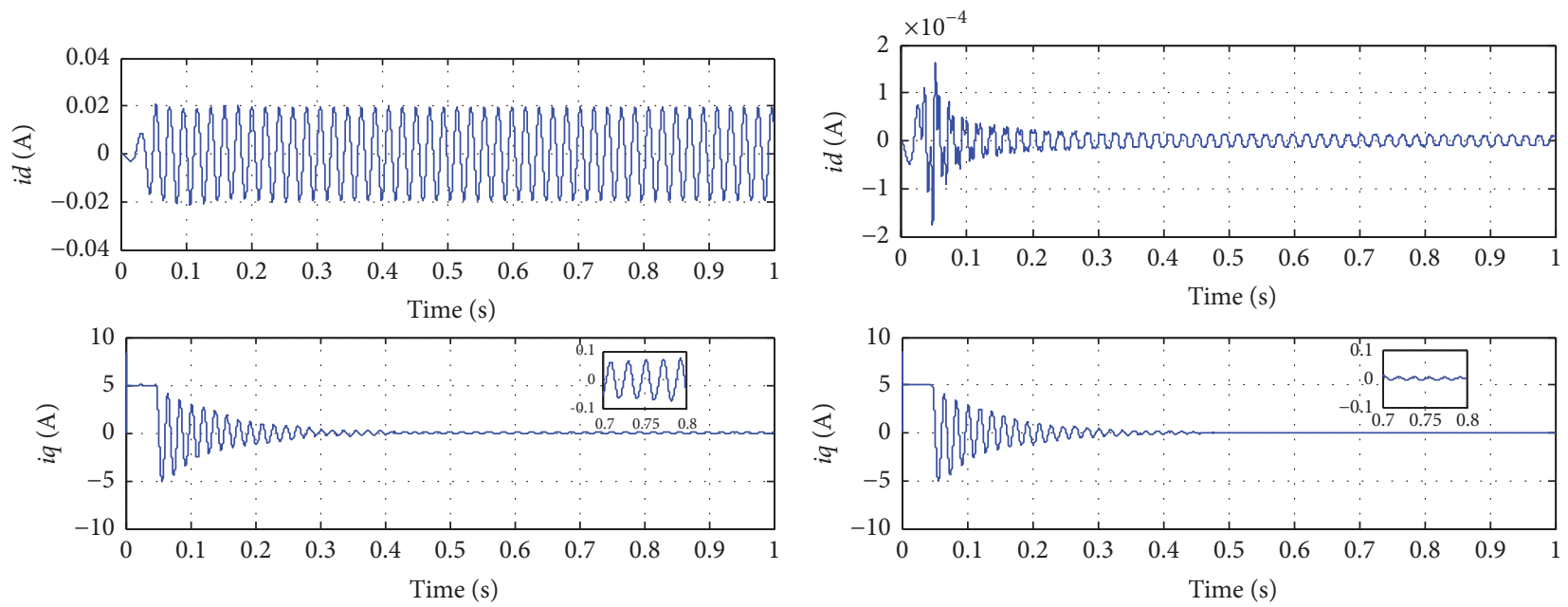

(a) Current waveforms before torque ripples minimization

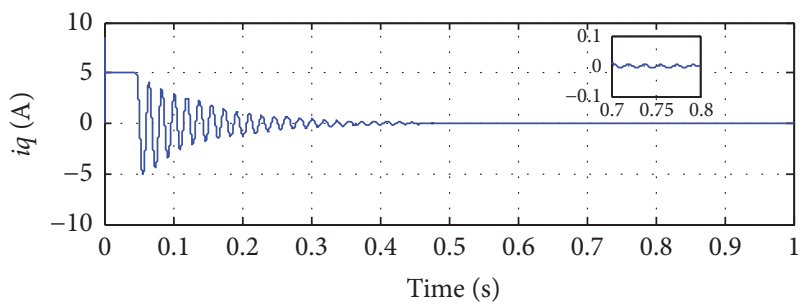

(b) Current waveforms after torque ripples minimization

FIGURE 7: Current waveforms before and after torque minimization.

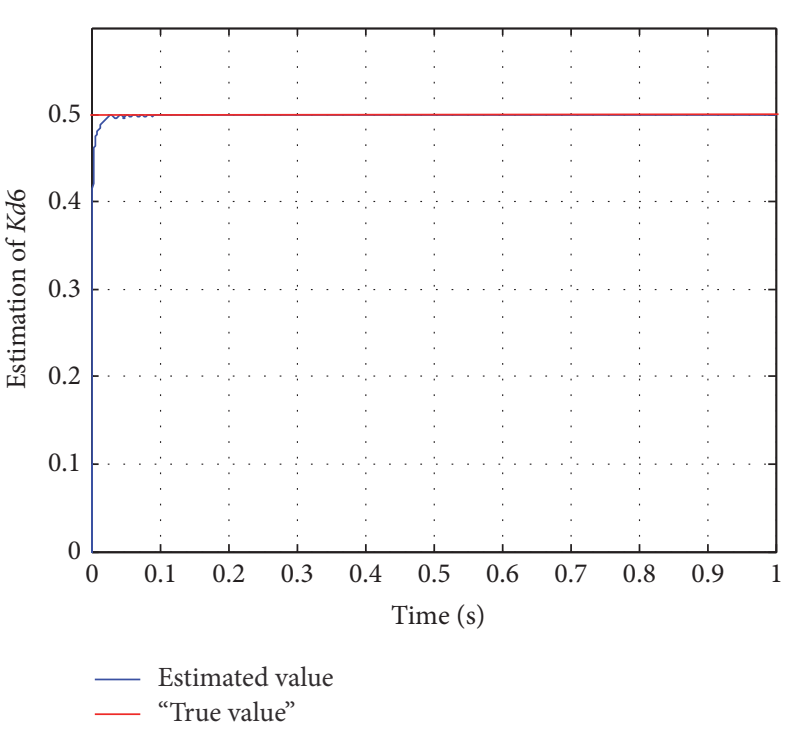

(a) Estimation of $\theta_{d}$
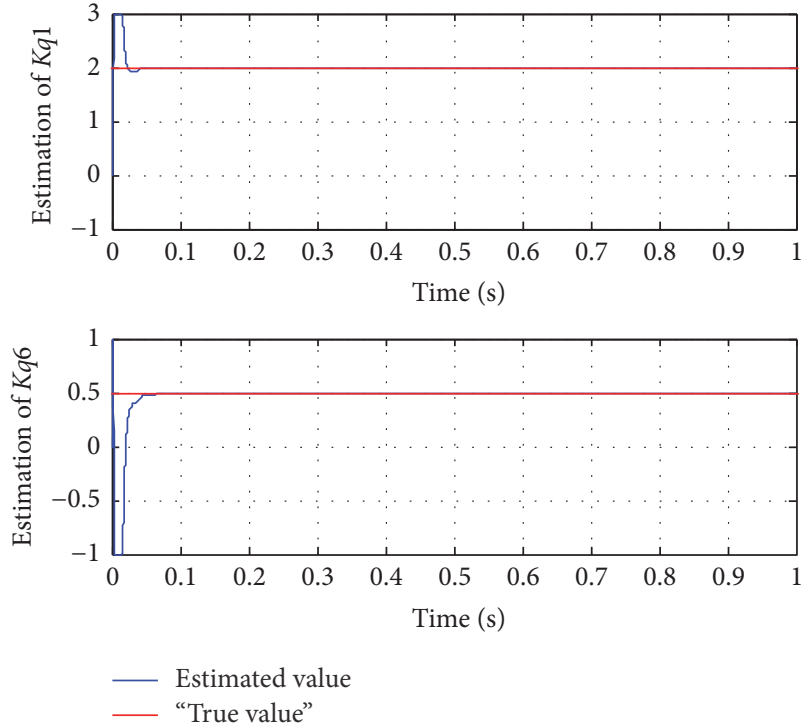

(b) Estimation of $\theta_{q}$

FIGURE 8: Estimated parameters of indirect adaption law.

\section{Conflicts of Interest}

The authors declare that there are no conflicts of interest regarding the publication of this paper.

\section{Acknowledgments}

This research work is partially supported by the National Natural Science Foundation of China (Grant no. 61773138). The authors would gratefully acknowledge the support of the National Natural Science Foundation of China.

\section{References}

[1] W. Cao, B. C. Mecrow, G. J. Atkinson, J. W. Bennett, and D. J. Atkinson, "Overview of electric motor technologies used for more electric aircraft (MEA)," IEEE Transactions on Industrial Electronics, vol. 59, no. 9, pp. 3523-3531, 2012.

[2] M. C. Chou, C. M. Liaw, S. B. Chien, F. H. Shieh, J. R. Tsai, and H. C. Chang, "Robust current and torque controls for PMSM driven satellite reaction wheel," IEEE Transactions on Aerospace and Electronic Systems, vol. 47, no. 1, pp. 58-74, 2011.

[3] W. Lina, X. Kun, L. De Lillo, L. Empringham, and P. Wheeler, "PI controller relay auto-tuning using delay and phase margin 
in PMSM drives," Chinese Journal of Aeronautics, vol. 27, no. 6, pp. 1527-1537, 2014.

[4] G. Feng, C. Lai, and N. C. Kar, "A closed-loop fuzzy-logicbased current controller for PMSM torque ripple minimization using the magnitude of speed harmonic as the feedback control signal," IEEE Transactions on Industrial Electronics, vol. 64, no. 4, pp. 2642-2653, 2017.

[5] H. Zhu, X. Xiao, and Y. Li, "Torque ripple reduction of the torque predictive control scheme for permanent-magnet synchronous motors," IEEE Transactions on Industrial Electronics, vol. 59, no. 2, pp. 871-877, 2012.

[6] J. Y. Hung and Z. Ding, "Design currents to reduce torque ripple in brushless permanent magnet motors," IEE Proceedings B: Electric Power Applications, vol. 140, no. 4, pp. 260-266, 1993.

[7] V. Petrović, R. Ortega, A. M. Stanković, and G. Tadmor, "Design and implementation of an adaptive controller for torque ripple minimization in PM synchronous motors," IEEE Transactions on Power Electronics, vol. 15, no. 5, pp. 871-880, 2000.

[8] W. Qian, S. K. Panda, and J. X. Xu, "Speed ripple minimization in PM synchronous motor using iterative learning control," IEEE Transactions on Energy Conversion, vol. 20, no. 1, pp. 5361, 2005.

[9] J.-X. Xu, S. K. Panda, Y.-J. Pan, T. H. Lee, and B. H. Lam, "A modular control scheme for PMSM speed control with pulsating torque minimization," IEEE Transactions on Industrial Electronics, vol. 51, no. 3, pp. 526-536, 2004.

[10] Z. Zhong, S. Jiang, Y. Zhou, and S. Zhou, "Active torque ripple reduction based on an analytical model of torque," IET Electric Power Applications, vol. 11, no. 3, pp. 331-341, 2017.

[11] B. Yao and M. Tomizuka, "Adaptive robust control of MIMO nonlinear systems in semi-strict feedback forms," Automatica, vol. 37, no. 9, pp. 1305-1321, 2001.

[12] Z. Chen, B. Yao, and Q. Wang, " $\mu$-synthesis-based adaptive robust control of linear motor driven stages with highfrequency dynamics: A case study," IEEE/ASME Transactions on Mechatronics, vol. 20, no. 3, pp. 1482-1490, 2015.

[13] J. Yao, Z. Jiao, and B. Yao, "Nonlinear adaptive robust backstepping force control of hydraulic load simulator: Theory and experiments," Journal of Mechanical Science and Technology, vol. 28, no. 4, pp. 1499-1507, 2014.

[14] S. Chen, Z. Chen, B. Yao et al., "Adaptive robust cascade force control of 1-dof hydraulic exoskeleton for human performance augmentation," IEEE/ASME Transactions on Mechatronics, vol. 22, no. 2, pp. 589-600, 2017.

[15] J. Yao, Z. Jiao, and D. Ma, "Adaptive robust control of dc motors with extended state observer," IEEE Transactions on Industrial Electronics, vol. 61, no. 7, pp. 3630-3637, 2014.

[16] Z. Chen, B. Yao, and Q. Wang, "Accurate motion control of linear motors with adaptive robust compensation of nonlinear electromagnetic field effect," IEEE/ASME Transactions on Mechatronics, vol. 18, no. 3, pp. 1122-1129, 2013.

[17] C. X. Hu, B. Yao, and Q. F. Wang, "Performance-oriented adaptive robust control of a class of nonlinear systems preceded by unknown dead zone with comparative experimental results," IEEE/ASME Transactions on Mechatronics, vol. 18, no. 1, pp. 178189, 2013.

[18] B. Yao and A. Palmer, "Indirect adaptive robust control of SISO nonlinear systems in semi-strict feedback forms," in Proceedings of the 15th World Congress of the International Federation of Automatic Control, vol. 35, pp. 397-402, Barcelona, Spain, 2002.
[19] A. Mohanty and B. Yao, "Indirect adaptive robust control of hydraulic manipulators with accurate parameter estimates," IEEE Transactions on Control Systems Technology, vol. 19, no. 3, pp. 567-575, 2011.

[20] G. Almandoz, J. Poza, M. Á. Rodríguez, and A. González, "Analytic model of a PMSM considering spatial harmonics," in Proceedings of the International Symposium on Power Electronics, Electrical Drives, Automation and Motion (SPEEDAM '08), pp. 603-608, Ischia, Italy, June 2008.

[21] N. Nakao and K. Akatsu, "Suppressing pulsating torques: Torque ripple control for synchronous motors," IEEE Industry Applications Magazine, vol. 20, no. 6, pp. 33-44, 2014.

[22] N. Zhou, J. W. Pierre, D. J. Trudnowski, and R. T. Guttromson, "Robust RLS methods for online estimation of power system electromechanical modes," IEEE Transactions on Power Systems, vol. 22, no. 3, pp. 1240-1249, 2007.

[23] S. J. Underwood and I. Husain, "Online parameter estimation and adaptive control of permanent-magnet synchronous machines," IEEE Transactions on Industrial Electronics, vol. 57, no. 7, pp. 2435-2443, 2010.

[24] M. M. Chansarkar and U. B. Desai, "A Robust recursive least squares algorithm," IEEE Transactions on Signal Processing, vol. 45, no. 7, pp. 1726-1735, 1997.

[25] T. Bonesky, "Morozov's discrepancy principle and Tikhonovtype functionals," Inverse Problems, vol. 25, no. 1, Article ID 015015, 11 pages, 2009. 


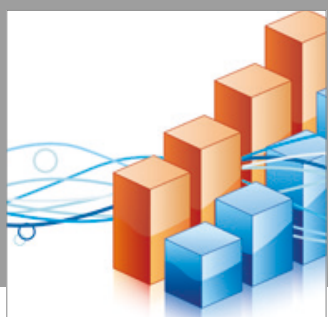

Advances in

Operations Research

vatersals

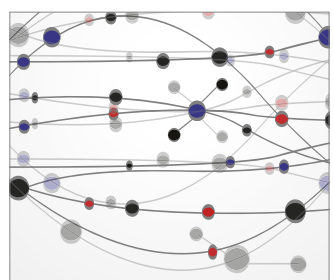

\section{The Scientific} World Journal
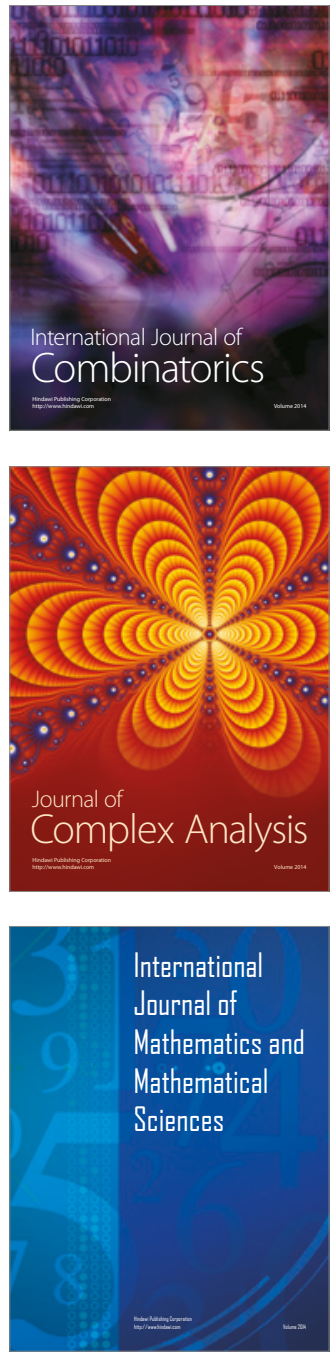
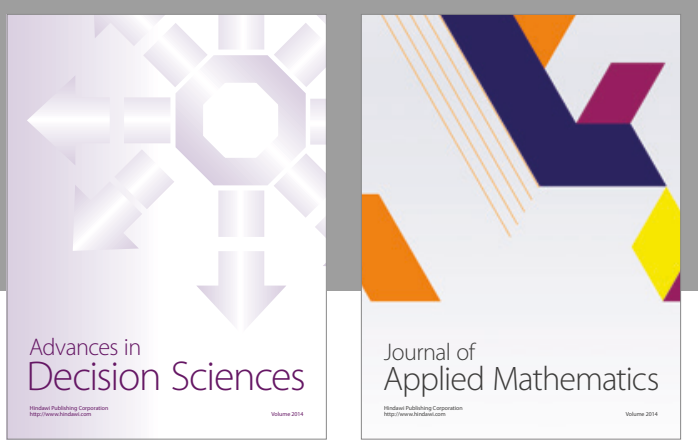

Algebra

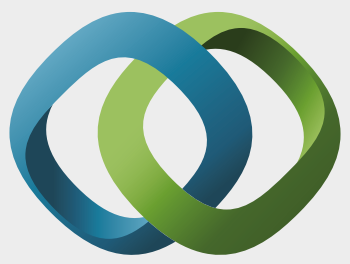

\section{Hindawi}

Submit your manuscripts at

https://www.hindawi.com
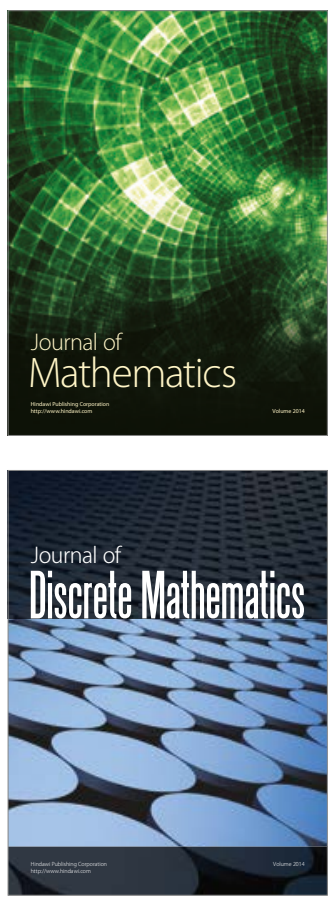

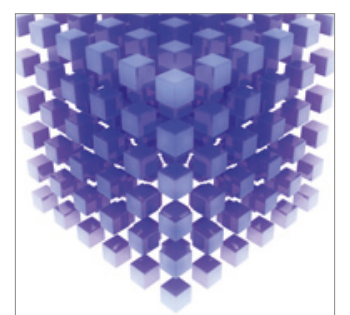

Mathematical Problems in Engineering
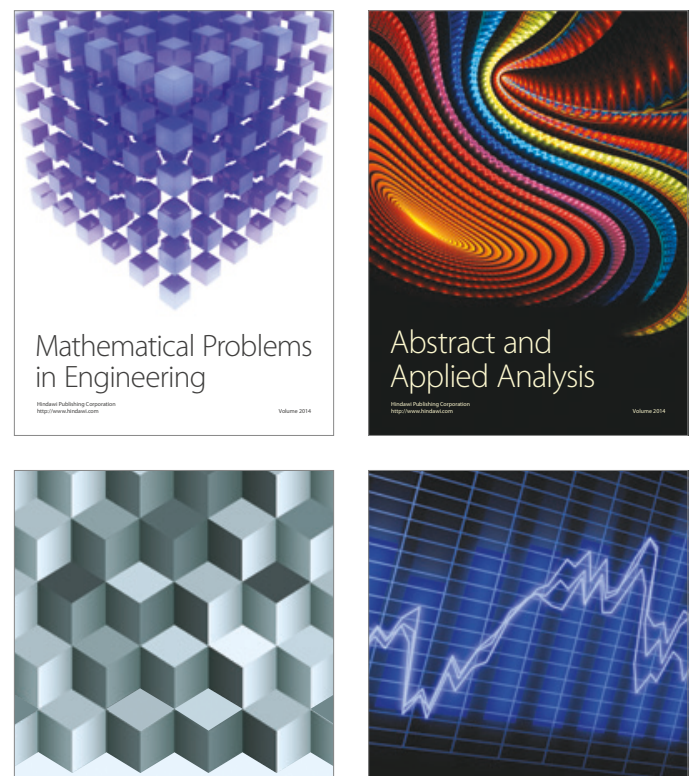

Journal of

Function Spaces

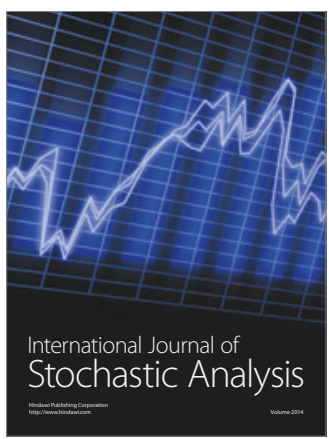

Probability and Statistics
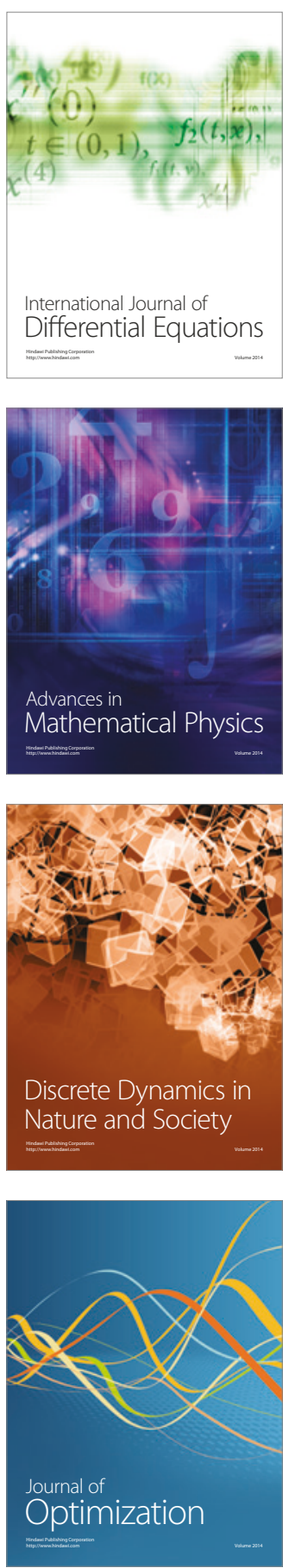